\title{
Mechanical behaviour of a sprayed concrete lining isolated by a sprayed waterproofing membrane
}

\author{
Masanari Nakashima ${ }^{\mathrm{a}, *}$, Anna-Lena Hammer ${ }^{\mathrm{b}}$, Markus Thewes ${ }^{\mathrm{b}}$, Mohammed Elshafie ${ }^{\mathrm{a}}$, Kenichi \\ Soga $\mathrm{a}^{\mathrm{a}}$ \\ ${ }^{a}$ Cambridge University Engineering Department, Cambridge, UK \\ ${ }^{b}$ Ruhr-University Bochum, Germany
}

Tel: +81-80-9085-7648 Email: nakashima.8a9.masanari@jp.nssmc.com

\section{ARTICLE INFO}

Article history:

Received

Received

Accepted

Keywords:

Sprayed concrete lining

Waterproofing membrane

4-point bending test

Eccentric compression test

\begin{abstract}
A waterproofing membrane has an important role in a sprayed concrete lined (SCL) tunnel. In this study, the mechanical behaviour of an SCL with a sprayed waterproofing membrane was investigated by laboratory testing. Two different types of tests were performed to examine the behaviour at different parts of an SCL tunnel linings around a tunnel junction. A four-point bending test was conducted to investigate the behaviour of tunnel linings under a large bending moment, which would represent a section near the opening of a tunnel junction. An eccentric compression test was conducted to investigate the behaviour of tunnel linings under a large compressive axial force (hoop stress) and a relatively small bending moment, which would represent a section at the opposite side of the opening of a tunnel junction. Both test results indicate that an SCL with a sprayed waterproofing membrane behaved as a composite section with very limited slip at the interfaces. Designing the primary lining, the waterproofing membrane and the secondary lining of an SCL tunnel as a composite section could have significant practical implications including time and cost savings.
\end{abstract}

\section{Introduction}

Sprayed concrete linings (SCL) are often employed to construct tunnels because of their distinctive characteristics which are summarised below [1]:

1) Sprayed concrete is a structural material that can be used as a permanent lining.

2) The material behaviour of sprayed concrete, which is initially soft but can withstand large strains at an early age, is suitable with the goal of a lining that permits a certain level of ground deformation.

3) The increase in stiffness and strength with age of sprayed concrete is also compatible with the need to control ground deformations.

4) Sprayed concrete linings can be used to form complicated shapes.

In particular, the fourth point is considered as a great advantage when a tunnel has a complicated geometry; for example, at tunnel junctions and cross-passages.
Waterproofing is considered to be an important issue for SCL tunnels since water leakage from/to tunnels could degrade the durability of structures and may eventually cause unacceptable ground settlements in the long term. A waterproofing membrane is therefore sometimes required. There are two types of waterproofing membrane: (i) a sheet waterproofing membrane and (ii) a sprayed waterproofing membrane. The latter was developed relatively recently and could be well used in situations with complicated geometries.

One of the issues related to a waterproofing membrane is what assumptions can designers reasonably make about the behaviour of the lining during the design stage. In practice, a sheet membrane is considered to have frictionless surfaces; thus a lining with a sheet waterproofing membrane is usually assumed to behave as a noncomposite structure [1]. In combination with a cast-in-place secondary lining this is the common construction method in conventional SCL tunnelling. For sprayed waterproofing membranes, several reports [2][3] describe that commercially available spray waterproofing membranes have enough adhesion to a lining so that it can be 
designed as a composite structure. However, the reports mainly discuss the properties of the sprayed membrane material; there is very limited information on the overall mechanical behaviour of an SCL with the sprayed waterproofing membrane.

A design consideration of whether a combination of a primary layer of sprayed concrete with a sprayed waterproofing membrane and a secondary layer of sprayed concrete can behave as a composite structure or not could have a significant influence on the total construction cost of SCL tunnels. The objective of this study is to investigate the mechanical behaviour of an SCL tunnel lining, which includes a layer of sprayed waterproofing membrane, by laboratory testing. Two different types of tests were performed to examine the behaviour at different parts of an SCL tunnel lining around a tunnel junction/crosspassage. A four-point bending test was conducted to simulate the behaviour of the SCL tunnel linings under a large bending moment, which would represent a section adjacent to the opening of a tunnel junction. An eccentric compression test was conducted to simulate the behaviour of the SCL tunnel lining under a large compressive axial force (hoop stress) and a relatively small bending moment, which would represent a section at the opposite side of the opening of a tunnel junction.

\section{Concept of laboratory tests}

The behaviour of tunnel linings, in particular at a tunnel junction, can be complex because of the complicated distribution of bending moment and axial compressive force, which are generated by ground and water pressure.

Figure 1 illustrates a typical distribution of the internal force in a tunnel lining around a tunnel junction. A relatively large bending moment can be generated near the opening of a main tunnel lining (section 1 in Figure 1). Hence, the behaviour of the tunnel lining around the opening can be dominated by the bending moment rather than the compressive axial force. On the other hand, a large compressive axial force with some degree of bending moment can be generated at a tunnel section around an opposite section of the opening (section 2 in Figure 1). Considering the above two different conditions of tunnel linings, two types of laboratory tests were conducted; (a) a 4-point bending test and (b) an eccentric compression test.
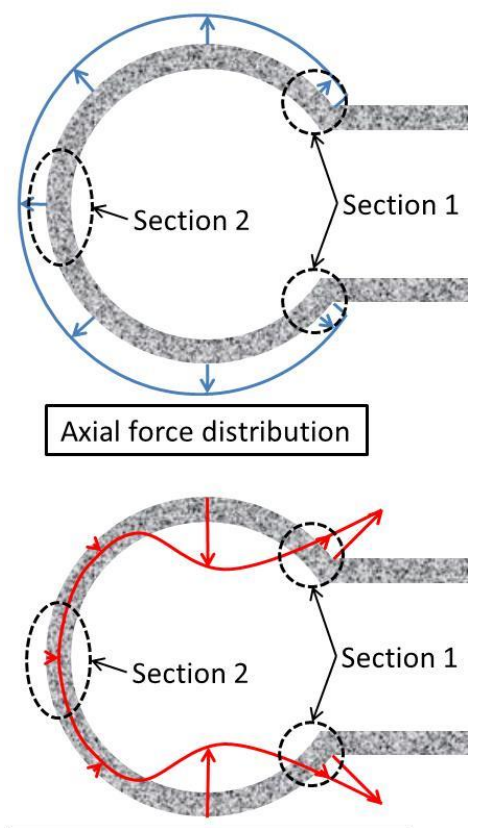

Bending moment distribution

Figure 1: Typical distribution of the internal force of the tunnel lining around a tunnel junction

A four-point bending test is often employed to investigate the behaviour of structures under bending moment. Since the bending moment between the two loading points is constant, the data interpretation can be straightforward.

To simulate an SCL tunnel section that is exposed to a certain level of bending moment, a plain sprayed concrete beam is not suitable since it has a small bending moment capacity. In this study, reinforcing bars were embedded into the tension side of the sprayed concrete beam as shown in Figure 2 to increase the bending moment capacity of the beam. In practice, this configuration may be different from the typical tunnel lining conditions where the secondary layer of the SCL may also have a layer of reinforcement. However, it was considered that this difference would not have a significant effect on the stress transfer through a waterproofing membrane.

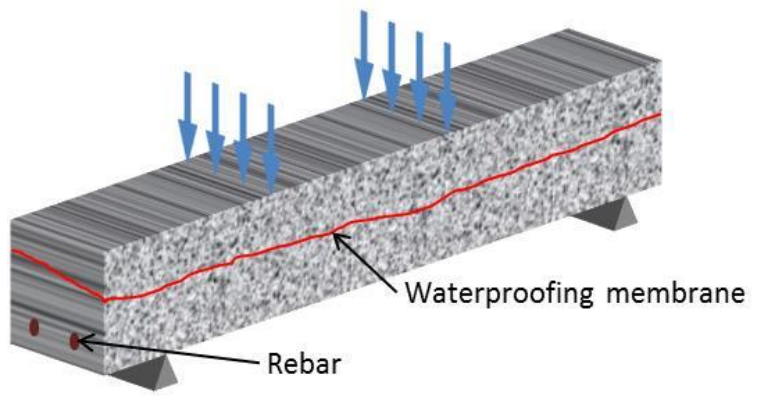

Figure 2: 4-point bending test 
A simple compression test on a column-shaped specimen could also be performed to investigate the behaviour under a large compressive axial force. A tunnel lining section around the opposite side of the opening would not only be exposed to compressive axial force but also be subjected to a relatively small bending moment as discussed before. In order to generate both, bending moment and compressive axial force, an eccentrically loaded compression test, shown in Figure 3 was performed.

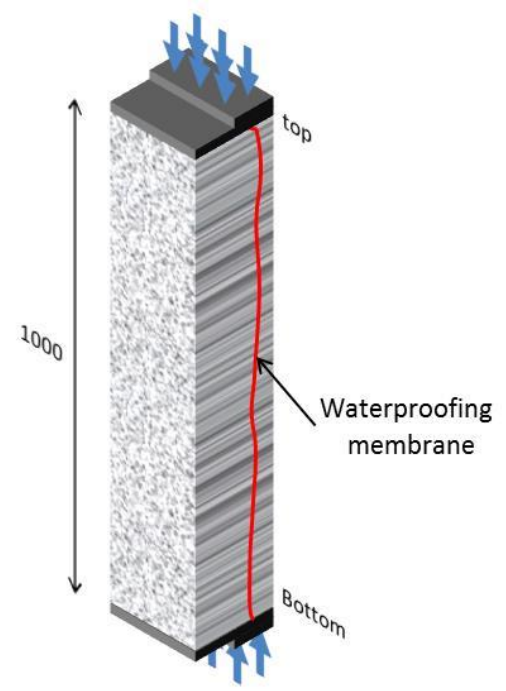

Figure 3: Eccentric compression test

Both tests were conducted with two specimens each, one with a sprayed waterproofing membrane and the other without, so that the two test results could be compared. A summary of the test cases conducted is given in Table 1.

It was assumed that the application of the sprayed waterproofing membrane could take place without any hindrance due to climatic conditions at the construction site. Further, no influence of moisture or water ingress during the application of the sprayed membrane was considered.

Table 1: Test case

\begin{tabular}{cccc}
\hline Case & Method & $\begin{array}{c}\text { Waterproofing } \\
\text { membrane }\end{array}$ & Reinforcement \\
\hline 1-A & 4-point bending & w/ & w/ \\
1-B & 4-point bending & w/o & w/ \\
2-A & Eccentric compression & w/ & w/o
\end{tabular}

\section{Methodologies of laboratory tests}

\subsection{Sample preparation}

All specimens were prepared by spraying a wet mix concrete using the robotic spraying machine available at Ruhr-University Bochum in order to represent similar conditions to a real tunnel lining situation [4]. This sprayed concrete testing rig allows for a minimisation of manual influences on the quality of the sprayed concrete, which could have a negative influence on the reproducibility of the results [5]. The SCL specimens (the properties of the sprayed concrete are shown in Appendix A) are prepared as follows:

1. A moistened wooden baseplate shown in Figure 4 was set vertically in front of the nozzle of the concrete spraying machine.

2. Ready-mixed concrete was transported from a local concrete plant to the laboratory.

3. The quality of the delivered concrete was checked in terms of workability and pumpability through slump testing and visual inspection.

4. If required, a small dosage of plasticiser was added to improve the workability and pumpability.

5. To create the first layer of the specimen, the concrete was sprayed through the nozzle as shown in Figure 5. The spraying was done using a robot, which followed a programmed pattern of movement including nozzle oscillations at a spraying distance of $1.0 \mathrm{~m}$, similar to typical field scenarios so that the concrete sprayed onto the baseplate would be as homogeneous as possible.

6. After the sprayed concrete has hardened reasonably (typically in one day), a waterproofing membrane (MASTERSEAL $^{\circledR}$ 345 by BASF) was then applied onto the surface of the sprayed concrete by a brush as shown in Figure 6. This product is usually sprayed by a special spraying machine on-site. However, according to the manufacturer, the final quality of the brushed product should be similar to that of the sprayed product. The material dried in a couple of hours and then it was applied repeatedly until the total thickness of the membrane was approximately $3 \mathrm{~mm}$ at the end. The thickness of the layer was measured using a needle-type thickness measuring device at a number of locations 
along the beam. It is acknowledged that this method of applying the sprayed waterproofing membrane might result in layering during testing, but the results, shown in Section 4 of the paper, indicated that insignificant or no layering has occurred during testing.

7. One day after applying the waterproofing membrane, the second layer of the sprayed concrete was created on top of the membrane using the concrete spraying robot.

8. During concrete curing, the surface of the specimen was covered by a wet fabric sheet to avoid excessive aridity.

9. When the concrete had hardened for one week, the sprayed concrete panel was cut into a beam and two columns with a diamond blade concrete cutter.

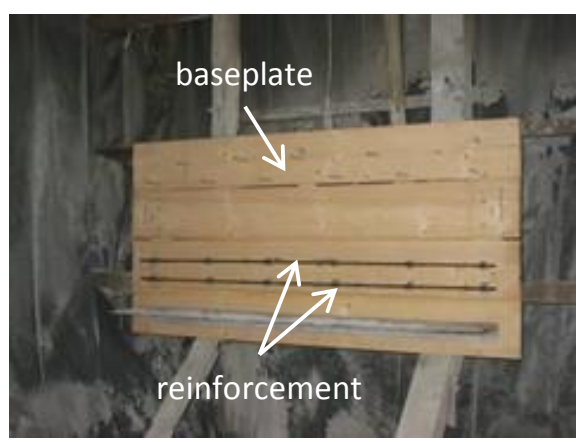

Figure 4: Setting of the baseplate with a pair of rebar

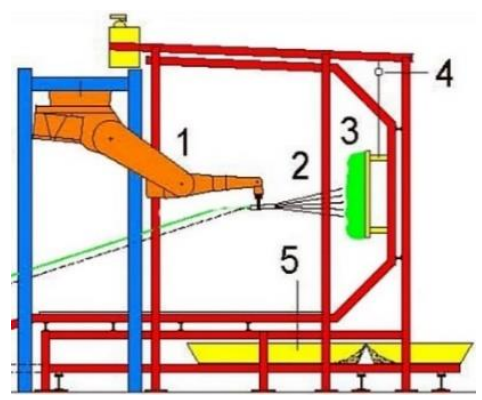

Figure 5: Sketch of the robotic sprayed concrete testing rig (1 robot, 2 nozzle, 3 baseplate with sprayed concrete, 4 weighing scale, 5 rebound collection)

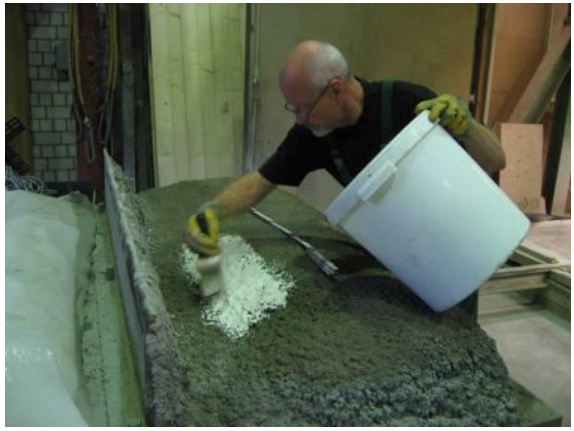

Figure 6: Applying the sprayable waterproofing membrane by brush as confirmed by manufacturer

\subsection{Four-point bending test}

\subsubsection{Specimen and instrumentation}

The dimensions of the SCL beam for the fourpoint bending tests (1-A and $1-\mathrm{B})$ were $1.2 \mathrm{~m} \mathrm{x}$ $0.2 \mathrm{~m} \times 0.2 \mathrm{~m}$. Strains of the concrete and rebar were measured by foil strain gauges, whereas vertical displacements of the beam were measured by LVDTs as shown in Figure 7. Loads were also recorded by using a load cell.
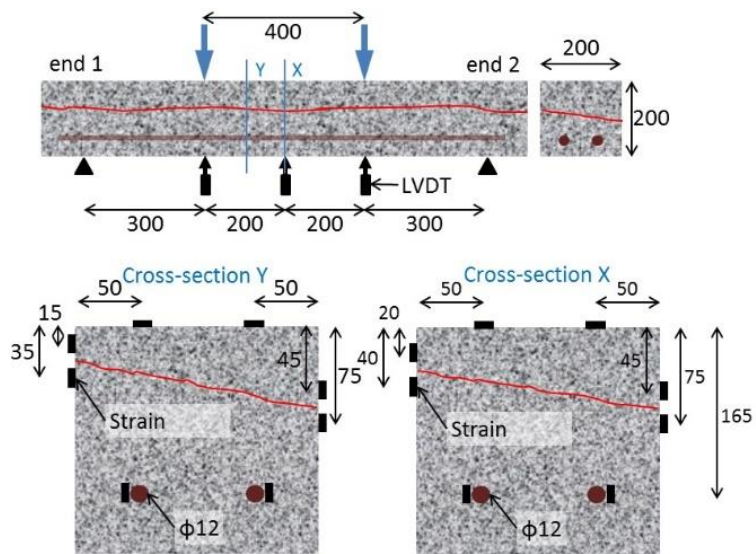

Figure 7: Beam specimen

The waterproofing membrane inclines in the specimen as Figure 7 shows. In practice, the surface of the membrane will be erratically inclined with respect to the bending axis due to the uneven nature of the sprayed concrete and, even more, due to the uneven nature of the substrate. The specimen accordingly has an inclined membrane in order to represent this fact in a qualitative way. Further, it is assumed that strain and shear stress in the specimen are on the conservative side in comparison with practice, because the substrate of the lining restrains dilation 
of the outer surface of the lining, which could increase the shear stiffness of the lining.

\subsubsection{Loading}

The test was performed by controlling the stroke of the hydraulic jack rather than the load, providing displacement controlled conditions. In order to avoid sudden failure after the yielding of the rebars, the speed of the hydraulic jack was set to $0.2 \mathrm{~mm} / \mathrm{min}$. Figure 8 shows the setting of the four-point bending test.

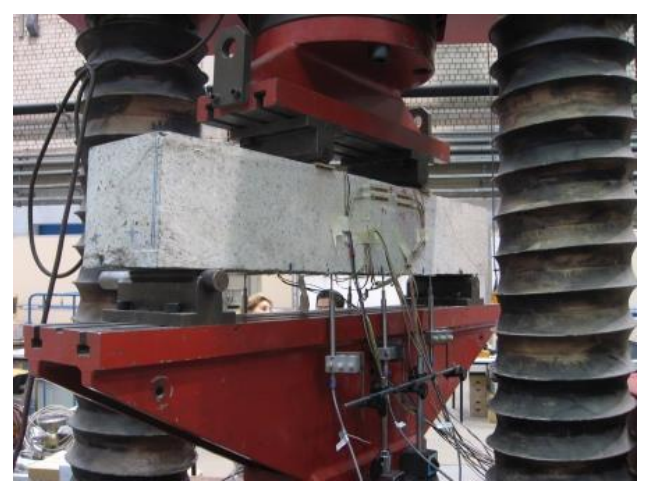

Figure 8: Setting of the four-point bending test (1-A)

\subsection{Eccentric compression test}

\subsubsection{Specimen and instrumentation}

The dimensions of the SCL columns for the eccentric compression tests (2-A and 2-B) were $1.0 \mathrm{~m} \times 0.2 \mathrm{~m} \times 0.2 \mathrm{~m}$. Strains of the concrete were measured by foil strain gauges attached at the surface as shown in Figure 9. The load was also measured by a load cell.

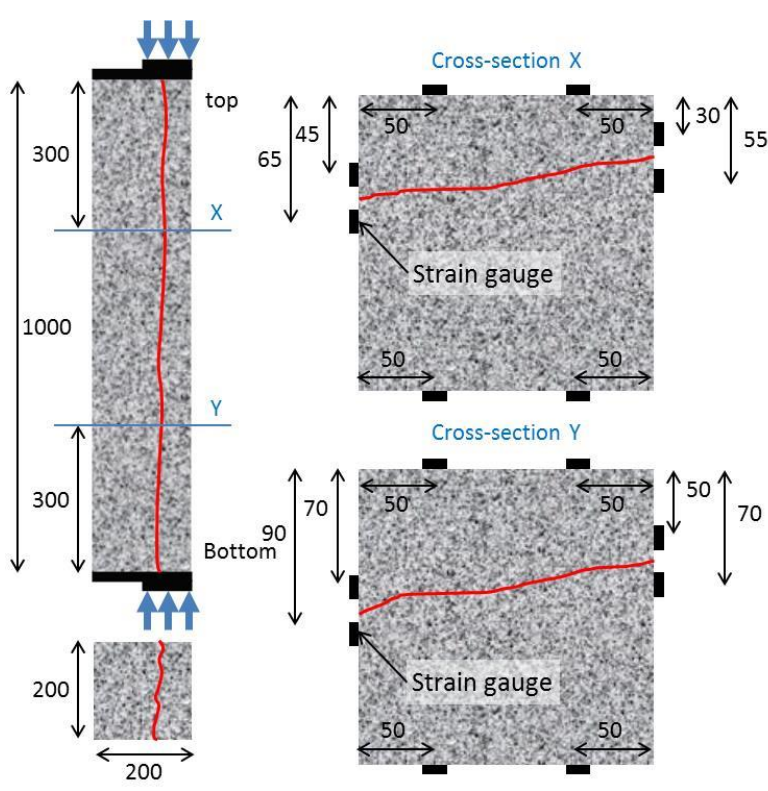

Figure 9: Column specimen

\subsubsection{Loading}

One of the critical aspects of the eccentric compression test is how to determine the amount of eccentricity and how to control it during testing. In order to investigate the shear stress transfer through a waterproofing membrane, a larger bending moment (i.e. larger eccentricity) could be more desirable, since the stress distribution corresponding to the bending moment in a crosssection of a specimen could be generated by shear stress transfer. However, an excessive eccentricity would generate an excessive bending moment, which might cause a brittle failure of the unreinforced specimens. Therefore, it was decided that the eccentricity of the test should not exceed one sixth of the height of the specimens, which was approximately $33 \mathrm{~mm}$.

An end steel plate, which was set between the hydraulic jack and the specimen, was designed to give a specific eccentric compression force to the columns. As shown in Figure 10, the end steel plate consisted of an upper plate and a bottom plate, and their dimensions were $100 \mathrm{~mm} \times 200 \mathrm{~mm}$ $\mathrm{x} 30 \mathrm{~mm}$, and $200 \mathrm{~mm} \times 200 \mathrm{~mm} \times 30 \mathrm{~mm}$, respectively. A soft wooden plate was installed between the bottom steel plate and the specimen to avoid stress concentrations at the ends. 


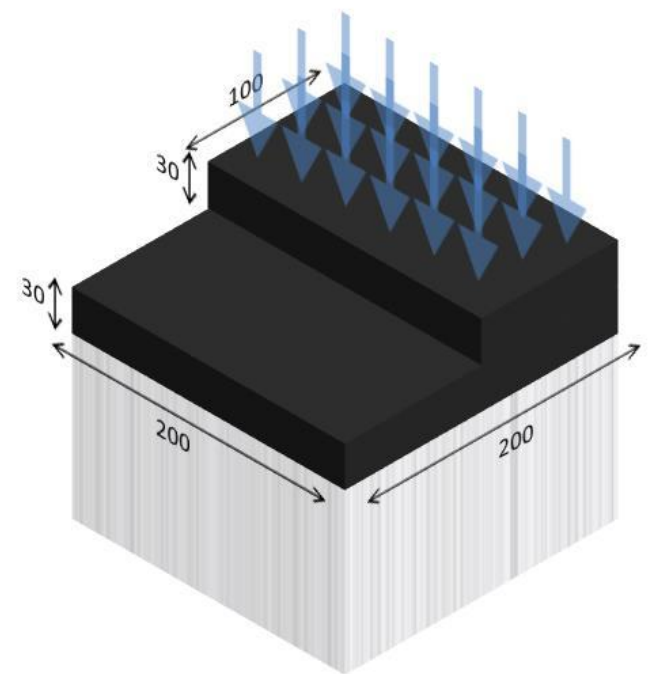

Figure 10: End plate for eccentric compression tests

Only the upper plate, which was set on one side of the bottom plate, would be subjected to the displacement of the hydraulic jack; this ensured that the compressive force is applied at a specific eccentricity to the specimen through the bottom plate, which covered the whole area of the end of the specimens as shown in Figure 10. One advantage of this approach was to avoid unnecessary stress concentrations at the end of the columns, which might cause a brittle failure. On the other hand, using this approach made it difficult to calculate the eccentricity precisely, since it was required to assume the angle of stress redistribution in the bottom plate. Assuming the angle of stress dispersion was $45^{\circ}$, the eccentricity was calculated as $35 \mathrm{~mm}$ as shown in Figure 11 .

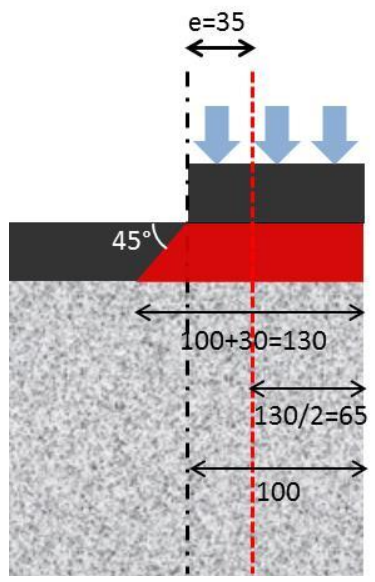

Figure 11: Predicted eccentricity

The setting of the eccentric compression test is shown in Figure 12. The tests were performed under the stroke control mode with $0.2 \mathrm{~mm} / \mathrm{min}$ speed, which is the same speed used for the fourpoint bending test.

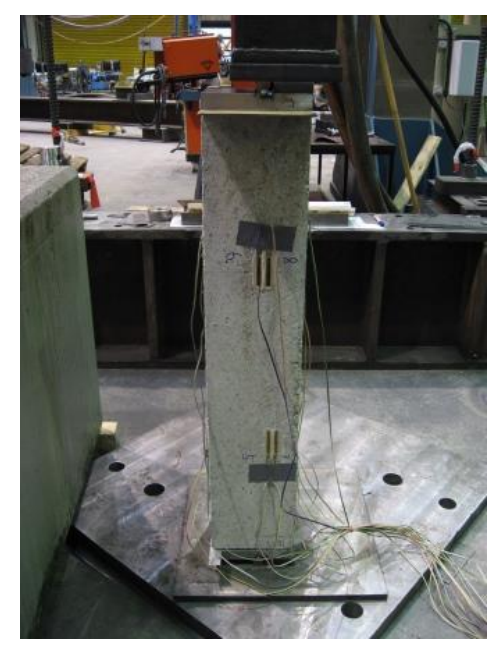

Figure 12: Setting of the eccentric compression test (2-A and 2-B)

\subsection{Cylindrical tests for data analysis}

Three cylindrical compression tests were also performed to evaluate the strength and the Young's modulus of the sprayed concrete in accordance with EN 206-1. Test specimens were cut from the same sprayed concrete panel and two foil strain gages were applied on the side of the specimens as shown in Figure 13. They were tested on the same day when the four-point bending test and the eccentric compression tests were conducted.

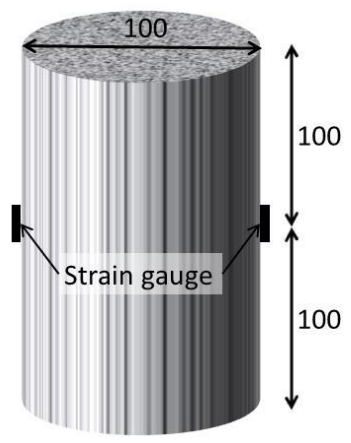

Figure 13: Cylinder test specimen

\section{$4 \quad$ Results and discussion}

\subsection{Strength and Young's modulus of the specimens}

The averaged strength and the Young's modulus of the three test samples, together with the standard deviation (the numbers in parentheses), are shown in Table 2. Those average values were used for 
analysing the test results, which would be discussed in a later section. Figure 14 shows the stress-strain curves obtained from the three samples.

Table 2: Strength and Young's modulus of sprayed concrete

\begin{tabular}{cc}
\hline $\begin{array}{c}\text { Strength } \\
(\mathrm{MPa})\end{array}$ & $\begin{array}{c}\text { Young's modulus } \\
(\mathrm{GPa})\end{array}$ \\
\hline $41.0(1.03)$ & $21(0.16)$ \\
\hline
\end{tabular}

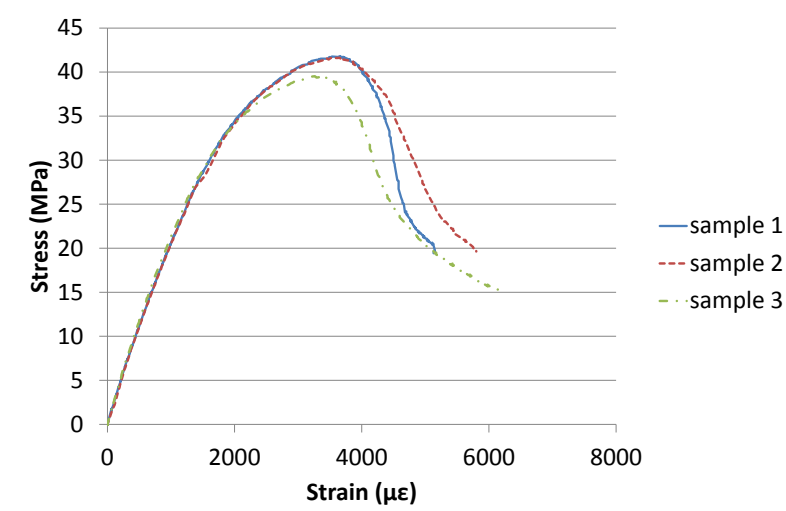

Figure 14: Stress-strain curves obtained from the three cylindrical compression tests

\subsection{Four-point bending test}

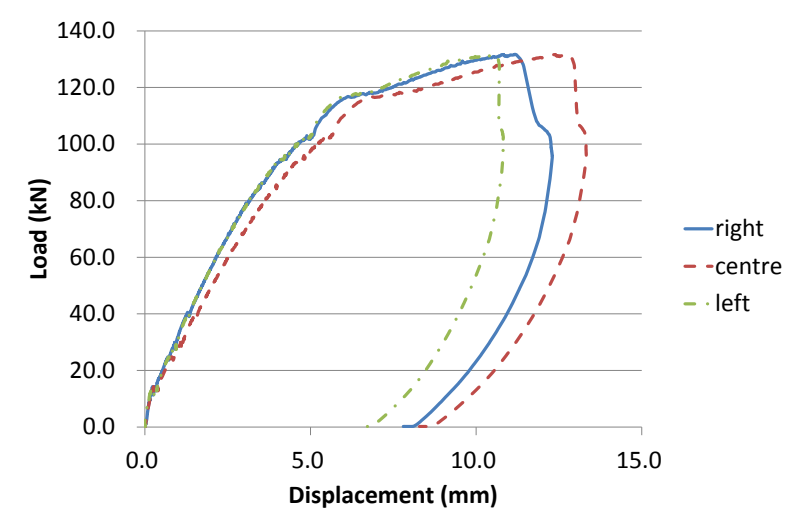

Figure 15 shows the developments of displacements of the beam at mid-span with loading of the test 1-A. The centre displacement was always larger than others although the right and the left displacements are not equal. It indicates that the beam was basically deformed as expected with slightly unbalanced force, which could be caused by imperfection of the specimens. Similar graphs can be plotted about the test 1-B. Nevertheless, it seems to be no problem to analyse the tests data as that expected bending moment was occurred in the beams.

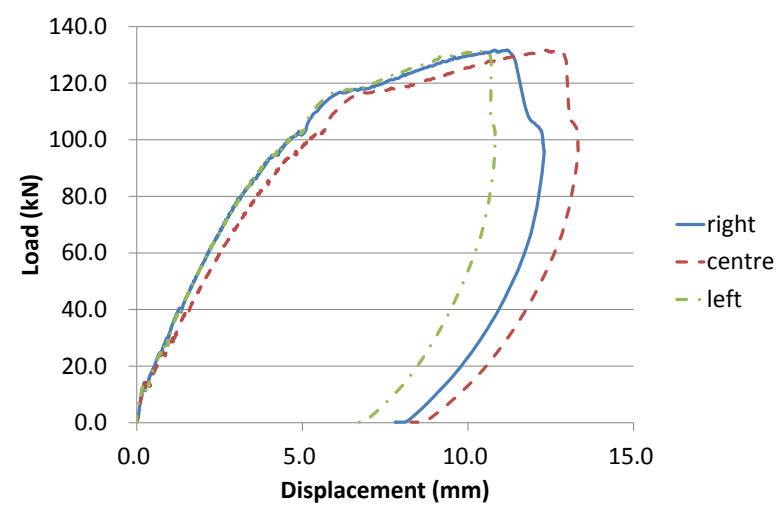

Figure 15: Relation between load and displacement of the beam at mid-span in the four-point bending test (1-A)

Figure 16 shows the relation between the bending moment and the curvature at the middle of the beam (i.e. section $\mathrm{X}$ in Figure 7) obtained from the 4-point bending tests. The bending moment and curvature were calculated from the load data and the strain data, respectively. The details of the calculation are shown in Appendix B. The slopes of the plots represents $E I$, which is the bending stiffness of the specimen, by considering Eq. (B.1). The "Theory (compound)" line in Figure 16 was drawn based on the assumption that the effective height of the beam was $200 \mathrm{~mm}$, which is the total height of the beam. On the other hand, the "Theory (separate)" line was drawn assuming that the effective height of the beam was $143 \mathrm{~mm}$, which is the averaged height of the bottom layer of the beam. The detail of the calculations of those "Theory" lines are shown in Appendix C.

Both the 1-A line and the 1-B line agreed with the "Theory (compound)" well until the behaviour of the beam dramatically changed as the rebars of the beam yielded. The gradient of the both lines at the beginning is bigger than that of "Theory (compound)" line. The reason of this difference is that the high stiffness of the beams before cracks are generated is not considered in "Theory" lines. A slight difference between the observed data and "Theory (compound)" line can be seen at high level bending moments $(16-18 \mathrm{kNm})$. This is probably due to the non-linear stress-strain relation of the sprayed concrete. Results show that the beam with a waterproofing membrane behaved as a compound beam. 


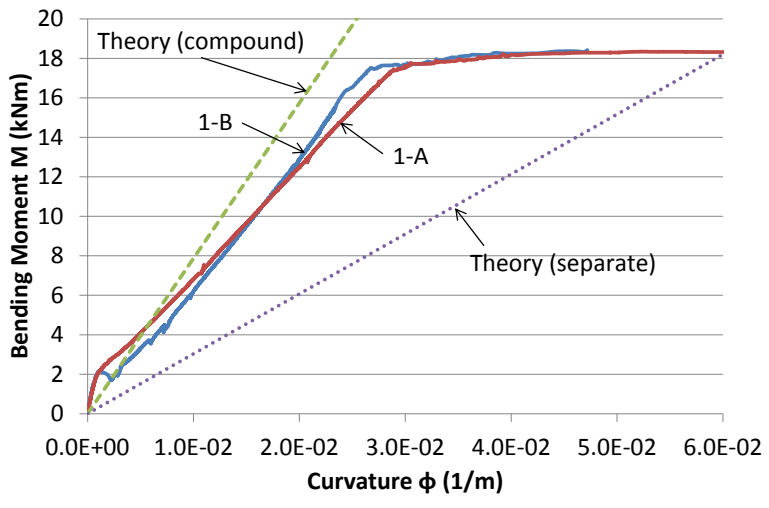

Figure 16: Relation between bending moment and curvature in the four-point bending test (1-A and 1-B)

Figure 17 shows the strain distribution at the centre of the beam (i.e. section $\mathrm{X}$ in Figure 7) at three different loads $(25 \%, 50 \%$ and $75 \%$ of the maximum load). The data from the strain gauges on the top of the beam were plotted on the zero line of the horizontal axis, whereas the data from the strain gauges attached on the reinforcements were plotted on the $145 \mathrm{~mm}$ line.

On the other hand, the strain data from the side strain gauges (see Figure 7) of 1-A test have a relatively large variation, even though some of them agree with the data of 1-B test. This variation in data might be caused by the rough surface of the beam. It was therefore difficult to have a clear evidence of the shear transfer through a waterproofing membrane. Nevertheless, it was concluded from the fact that the top and bottom strain data of 1-A test agreed with that of 1-B test that the beam with a waterproofing membrane generally behaved as a compound beam.

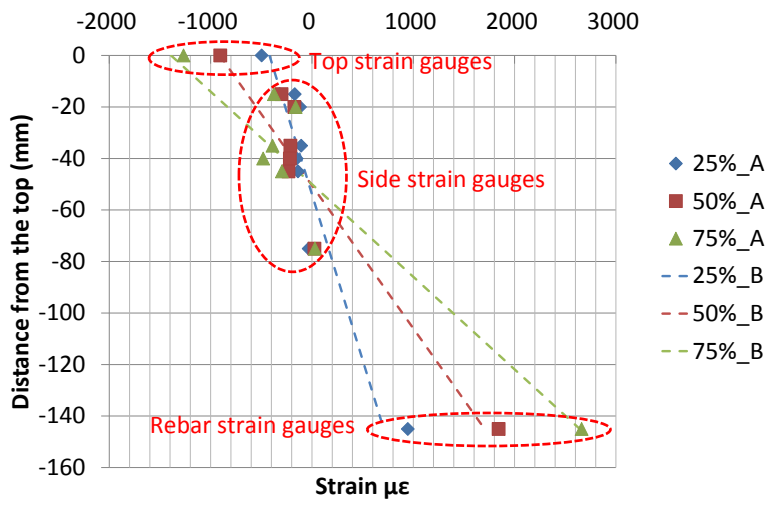

Figure 17: Strain distribution in the four-point bending test (1-A and 1-B)

\subsection{Eccentric compression test}

Figure 18 shows the strain-load history from the two eccentric compression tests. Large compressive strains were observed on the side of which the load was applied and small tensile strains were observed at the opposite side, confirming that an eccentric loading was applied to the specimens as expected.
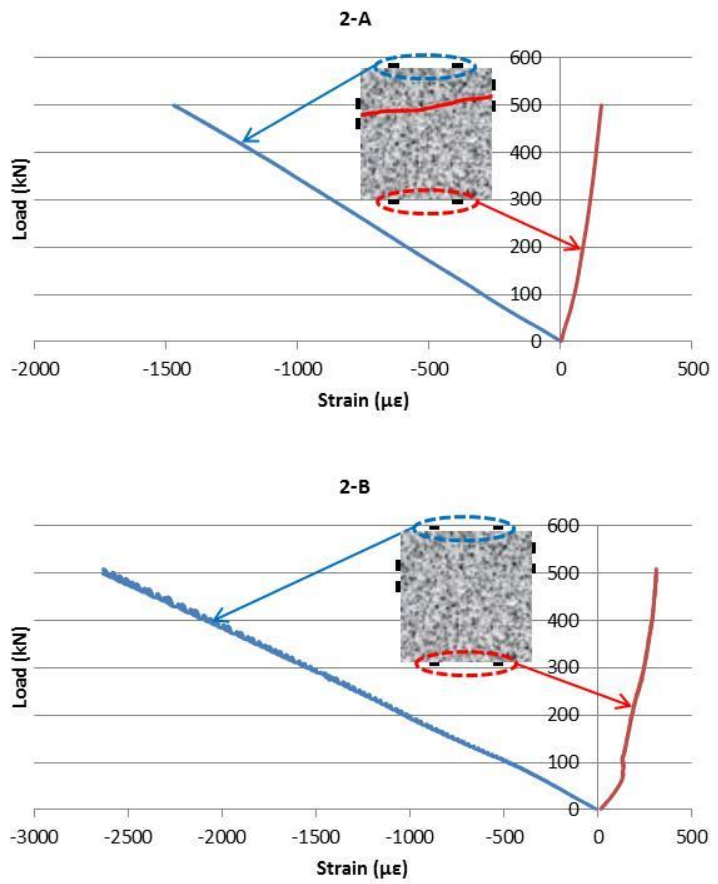

Figure 18: Strain history in the eccentric compression test (2-A and 2-B)

A theoretical relationship between eccentricity $e$ and strain data can be computed as shown in Appendix D. Figure 19 shows the changes in eccentricity with increasing load of the two tests. At smaller load levels, the values of the eccentricity were different between the two tests because they were influenced by the instability of the specimens and the accuracy of strain gauges. However, at larger load levels, the eccentricity values converged to a similar value of approximately $41 \mathrm{~mm}$, which was reasonably close to the value of $35 \mathrm{~mm}$ estimated earlier. If the column with a waterproofing membrane behaved as two separated columns, then each layer would have had a different eccentricity, generating a totally different strain distribution in a crosssection. 


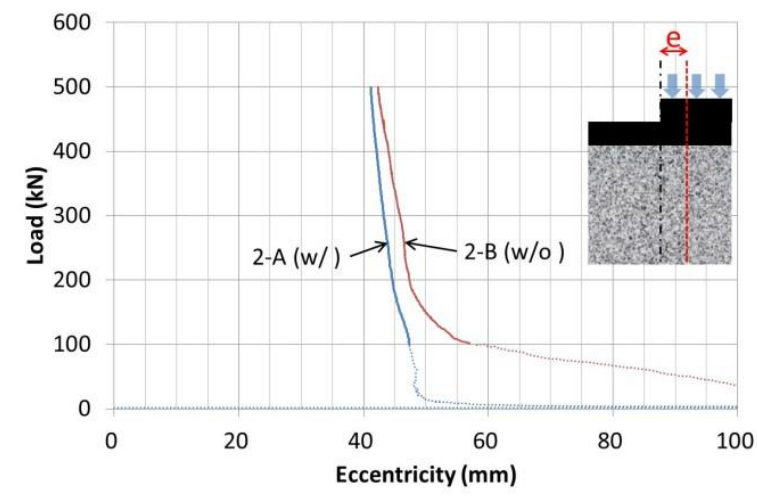

Figure 19: Observed eccentricities (2-A and 2-B)

In theory, a tilted stress distribution can be observed in only one direction corresponding to the eccentricity. However, in practice, the loading plate was slightly tilted in a direction perpendicular to the original eccentric direction due to non-perfectly levelled surface of the columns, which caused the behaviour of the two columns to be different, as shown in Figure 20. Therefore, in order to compare the data of the two specimens more carefully, it was required to do some compensation of both data that would remove the effect of strain generated by the additional unintended eccentricity.

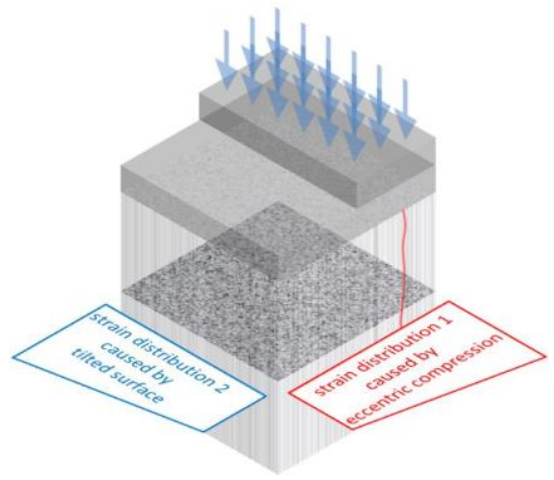

Figure 20: Schematic view of biaxial strain distribution
The method to remove the effect of strain distribution caused by the imperfection in the direction perpendicular to the eccentric loaded direction was done using Bernoulli-Euler theory and the details of the compensation are described in Appendix E.

The strain distributions at two sides (left and right in Figure 9) at two cross-sections (X: 300mm from the top, and $\mathrm{Y}: 300 \mathrm{~mm}$ from the bottom as shown in Figure 9) are shown in Figure 21. The strain data presented are those after compensating the raw strain data by removing the tilt effect in the perpendicular direction. The results are plotted at three different loads $(25 \%, 50 \%$ and $75 \%$ of the maximum load). Results show, for a given load, the strain distribution of a specimen with membrane (test 2-A) agrees well with that of a specimen without membrane (test 2-B). The data set at the right side of the cross-section $\mathrm{Y}$ gave a much large value after the $50 \%$ of the maximum loading and it is suspected that these strain gauges were faulty. However, the strains measured along the sides of Column A changed continuously and hence it appears that the shear stress are transferred through the waterproofing membrane. In summary, the results indicate that the specimen with a waterproofing membrane (Column A) behaved as a compound column. 

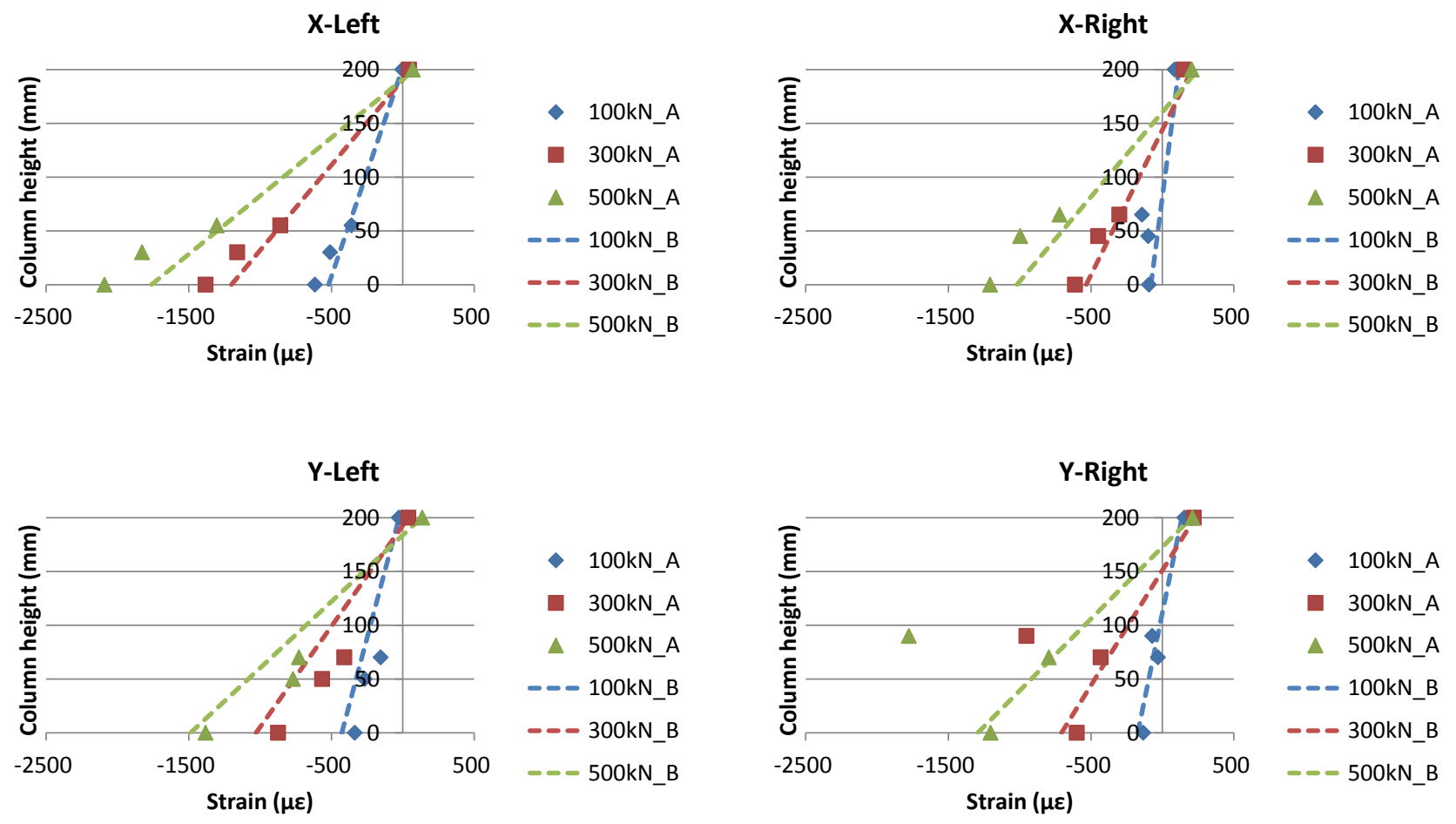

Figure 21: Strain distribution in the eccentric compression tests (2-A and 2-B)

\section{Conclusions and Outlook}

Laboratory tests were conducted to examine the mechanical behaviour of a sprayed concrete lining isolated by a sprayed waterproofing membrane. The loading applied to these tests was designed to simulate the predominant loading conditions expected in the vicinity of a tunnel junction. The findings are summarised as follows;

1. The four-point bending test (test 1-A and 1B);

- The stiffness, which were evaluated from the observed strain, indicated that the beam with a waterproofing membrane behaved as a compound beam under bending.

- The side strain gauges could not capture the shear stress transfer through a waterproofing membrane. This was mainly due to the resolution of strain gauge as well as the issue of attaching strain gauges on the rough surface of the beam. Further work is needed to improve this.

2. The Eccentric compression tests (tests 2-A and 2-B)

- At large loading conditions, the eccentricity measured in the column with a waterproofing membrane (Column A) was similar to that without a membrane (Column B). This suggests that Column $\mathrm{A}$ is behaving as a compound column under combined bending and axial compression.

- The strain distributions after compensating for the loading imperfection observed in the two tests showed Column A is exhibiting a compound column behaviour. Furthermore, shear stress transfer could be observed from the strain gauge data.

Designing the primary lining, the waterproofing membrane and the secondary lining of a SCL tunnel as a composite section could have significant practical implications. Although it is not possible to generalise any conclusions because of the limited amount of testing performed, the evidence of the significant load sharing between the sprayed concrete layers and the waterproofing membrane shown in this paper could open the path to further optimise the thicknesses of linings and potentially realise significant cost and time savings. Further testing and investigation are required in this area.

In addition to the structural behaviour, which was analysed in this study, a sprayed waterproofing membrane needs to have a durability similar to 
that of a sprayed concrete, a quality-controlled application process, and a verification of the full bond between sprayed membrane and concrete. These requirements were not part of the experiments presented here and should be part of further studies.

\section{Acknowledgements}

The authors would like to acknowledge the great support offered by technicians at the structural testing laboratory of Ruhr-University-Bochum.

\section{Appendix A}

The properties of the sprayed concrete are shown in Table A.1.

Table A.1: Properties of the sprayed concrete

\begin{tabular}{ccccc|c|c|c}
\hline \multirow{2}{*}{$\mathrm{W} / \mathrm{C}$} & $\mathrm{S} /(\mathrm{S}+\mathrm{G})$ & \multicolumn{6}{c}{$\mathrm{kg} / \mathrm{m}^{3}$} \\
& & $\begin{array}{c}\text { Water } \\
\text { (W) }\end{array}$ & $\begin{array}{c}\text { Cement } \\
(\mathrm{C})\end{array}$ & $\begin{array}{c}\text { Sand } \\
(\mathrm{S})\end{array}$ & $\begin{array}{c}\text { Gravel } \\
(\mathrm{G})\end{array}$ & \multicolumn{2}{c}{ Admixture } \\
\hline $45 \%$ & $60 \%$ & 193 & 430 & 1019 & Plasticiser & Retardant \\
\hline
\end{tabular}

The Young's modulus of the rebar is $205 \mathrm{GPa}$, and the yield strength of the rebar is $500 \mathrm{MPa}$ in test $1-\mathrm{A}$.

\section{Appendix B}

The bending moment at the middle of the beam in a four-point bending test, as shown in Figure B.1 below, can be calculated as follow;

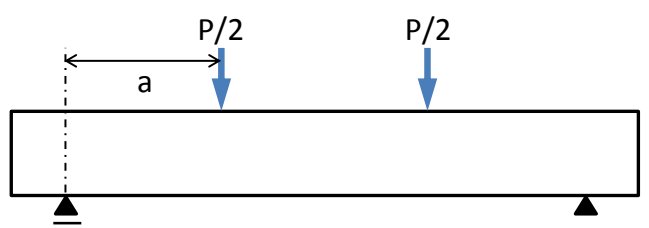

Figure B.1: Simply supported beam with constant bending moment between the two concentrated loads

$$
M=P a / 2
$$

where, $P$ is the load and $a$ is the distance between the load point and the support point, which was $300 \mathrm{~mm}$ in $1-\mathrm{A}$ and $1-\mathrm{B}$ tests.

A curvature can be calculated from the observed strains using the following equation.

$\phi=\left(\varepsilon_{\text {rebar }}-\varepsilon_{\text {top }}\right) / d$

where, $\varepsilon_{\text {rebar }}$ and $\varepsilon_{\text {top }}$ are the strain data of rebar and that of the top surface of the beam, respectively, and the $d$ is the distance from the top of the beam to the rebar.

\section{Appendix C}

The theoretical relation between bending moment and curvature on the basis of Bernoulli-Euler theory can be shown as below;

$$
M=E I \phi
$$

where, $I$ is the second moment of inertia of the beam, which is shown in Figure 7.

Assuming that concrete cannot withstand tensile force, the second moment of inertia can be calculated as follow;

$$
\begin{aligned}
I= & B x^{3} / 3+(d-x)^{2} n A_{s} \\
\quad x & =\left(\sqrt{2 n p+(n p)^{2}}-n p\right) d
\end{aligned}
$$

where, $B, x, d, n, A_{s}$ and $p$ are the height of the beam, the distance between neutral axis and the top edge of the beam, the effective height of the beam, the ratio of the Young's modulus of rebar and concrete, the total area of rebar, and the reinforcement ratio of the beam, respectively, as shown in Figure C.1 and Figure C.2. In "Separate" case, only the area below the membrane is valid since there is no reinforcement, which can withstand tensile force, above the membrane.

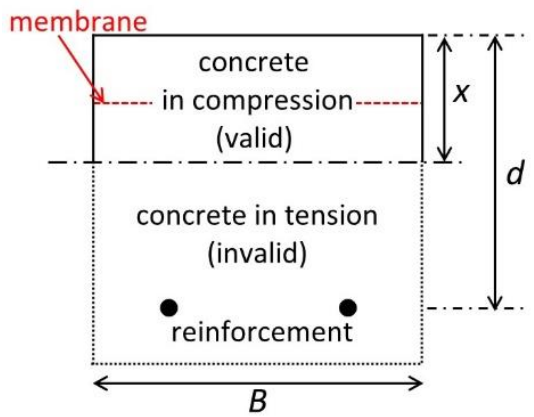

Figure C.1: Cross-section of the beam (Compound)

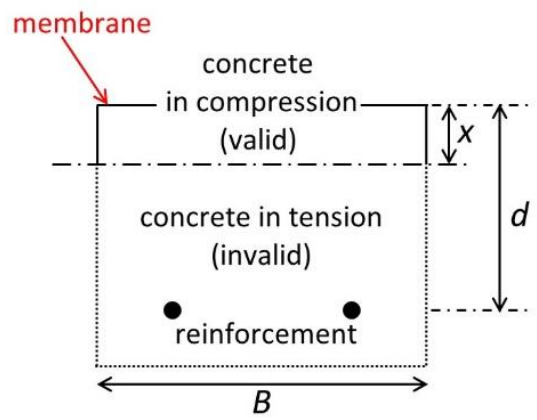

Figure C.2: Cross-section of the beam (Separate)

\section{Appendix D}


The definition of an eccentricity $e$ can be expressed by bending moment $M$ and axial force $N$ as shown below;

$$
e=M / N
$$

In addition, bending moment and axial force can be calculated from the observed strains as follows;

$$
\begin{array}{ll}
M=Z \sigma_{M}, & N=A \sigma_{N} \\
Z=b h^{2} / 6 & , \quad A=b h \\
\sigma_{M}=E \varepsilon_{M}, & \sigma_{N}=E \varepsilon_{N}
\end{array}
$$

where $Z$ is the module of the section and $A$ is the area of the columns, which can be calculated from the height $h$ and the width $b$ of the columns.

$\varepsilon_{M}$ and $\varepsilon_{N}$ are the strains which are caused by bending moment and axial force, respectively, and they can be calculated from the observed strains.

Substituting Eq. D.2 to Eq. D.1, the relation between an eccentricity and strains can be derived as follows;

$$
e=h \varepsilon_{M} / 6 \varepsilon_{N}
$$

\section{Appendix E}

The strain at the corner of the specimens can be calculated using the Bernoulli-Euler theory as shown in Figure D.1.

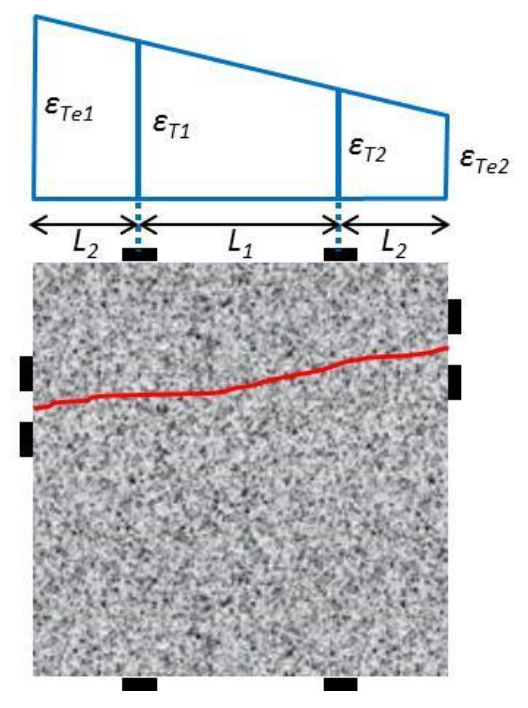

$$
\begin{aligned}
& \varepsilon_{T e 1}=\varepsilon_{T 1}+\frac{\varepsilon_{T 1}-\varepsilon_{T 2}}{L_{1}} L_{2} \\
& \varepsilon_{T e 2}=\varepsilon_{T 2}-\frac{\varepsilon_{T 1}-\varepsilon_{T 2}}{L_{1}} L_{2}
\end{aligned}
$$

\section{References}

[1] A. Thomas, Sprayed Concrete Lined Tunnels. London: Taylor \& Francis, 2009.

[2] R. Makhlouf and K. Holter, "Rehabilitation of concrete lined tunnels using a composite sprayed liner with sprayed concrete and sprayable waterproofing Membrane; The Chekka Road", ITA World Tunnel Congress, Agra, India, pp. 1175-1182, 2008.

[3] K. Holter, R. Bridge, and O. Tappy, "Design and Construction of Permanent Waterproof Tunnel Linings Based on Sprayed Concrete and Spray-Applied Double-Bonded Membrane," ... Int. Conf. Underground Construction - Transport and City Tunnels, Prague, Czech Republic, pp. 121-126, 2010.

[4] B. Maidl, M. Thewes, and U. Maidl, Handbook of Tunnel Engineering I, 1st ed. Berlin: Ernst und Sohn, 2013.

M. Thewes and G. Vollmann, "Applications of a shotcrete robot for creating an immediate comparability of mix-designs", ITA World Tunnel Congress, Prague, Czech Republic, pp. 1377-1382, 2007.
Figure E.1: Strain compensation for the eccentric compression tests

$\varepsilon_{T e l}$ and $\varepsilon_{T e l}$ can be given as follows; 